\title{
Research on liability Identification System of Road Traffic Accident
}

\author{
Sai Liu, Zhen-Jiang Zhang*, Zi-Hang Yu
}

\begin{abstract}
Beijing Key Laboratory of Communication and Systems, School of Electronic and Information Engineering, Beijing Jiaotong University, Haidian District, Beijing, China

\{21111052, zhangzhenjiang, 21120163\}@bjtu.edu.cn
\end{abstract}

\begin{abstract}
With the rapid development of social economy and the increasing improvement of people's living standards, car ownership is increasing exponentially, and road traffic accidents occur frequently. The identification of liability of traffic accident is an important problem in accident handling, which relates to the life and property interests of the litigants concerned. At present, the identification of liability of traffic accident basically depends on the manual judgment of law enforcement department, which is influenced by the experience and human feelings of law enforcement personnel, and has certain randomness and uncertainty. This paper proposes a traffic accident intelligent responsibility identification system based on Case-based Reasoning and D-S Evidence Theory. In the process of liability identification, Case-based Reasoning and D-S Evidence Theory are combined to analyze cases in case database. According to the case similarity, the basic probability of the litigants bearing the main liability is fused to obtain the probability interval of each litigant bearing the main liability, so as to determine the main liability person of the accident. By testing in real cases, the method proposed in this paper has achieved outstanding effect in identifying the main liability of traffic accidents.
\end{abstract}

Keywords: liability identification, case-based reasoning, D-S evidence theory, traffic accident

\section{Introduction}

In recent years, with the rapid development of national economy, the number of private cars has increased significantly. Road traffic activities have become more and more frequent, which has resulted in a significant increase in the number of traffic accidents, and the deterioration of road traffic safety situation. Traffic safety management, especially traffic accident handling, is facing severe challenges. According to statistics, there are more than 200,000 road traffic accidents causing casualties and more than 8 million minor accidents causing property damage every year. The handling of every traffic accident is closely related to the vital interests of the litigants involved. The identification of traffic accident liability concerns the legitimate rights and interests of the litigants, social equity and justice and the credibility of law enforcement agencies. According to the content of existing laws and regulations, the traffic accident treatment flow includes: scene investigation, liability identification, responsible person punishment, accident damage compensation and mediation, etc [1]. In the process of accident handling, one of the most core links is the identification of traffic accident liability.

The identification of traffic accident liability is the expression of the relationship between the fault behavior of the litigants involved in the traffic accident and the traffic accident and their obligations. In essence, the identification of traffic accident liability is the confirmation of the influence of the illegal behavior of the litigants involved in the traffic accident in the formation of traffic accident damage [2]. It is a qualitative and quantitative description of the cause of the accident, the specific quantification of the nature and circumstances of the violation of laws and regulations by the litigants concerned, and an important basis for determining the type and severity of punishment to the litigant concerned.

But there are still some problems in the identification of liability of road traffic accidents. On the one hand, the occurrence of road traffic accidents is unpredictable, and the causes of accidents are complicated and composed of many litigants [3]. In the analysis of the relationship between the fault behavior of the litigants and the liability of traffic accidents, the unilateral fault shows less causality, but more of the mixed fault shows co-causal relationship, which has strong uncertainty [4-5]. On the other hand, the knowledge system of traffic accident handling personnel in law, technology and other aspects may not be perfect, and the judgment of accident liability relies too much on empirical judgment, which cannot guarantee the objectivity and scientificity of liability identification to a certain extent.

Therefore, for many years, relevant scientific research departments have been studying and applying modern technologies (such as artificial intelligence, expert systems) to investigate and deal with traffic accidents. With the continuous development and improvement of computer application technology, the use of artificial intelligence 
and expert system to help law enforcement personnel to deal with road traffic accidents has become a reality, and more and more shows its superiority.

The acquisition of "knowledge" has hindered the development of expert system. Case-based Reasoning (CBR) uses existing successful cases and expert experience to reflect knowledge in the form of cases and solves the difficult problem of knowledge acquisition. With the development of artificial intelligence, CBR system has become increasingly mature in research and application, and has a very wide range of applications. Different from the traditional Rule-based Reasoning (RBR) system, knowledge does not need to be regularized. Instead, it learns from the way humans deal with problems, directly stores the previous experience and solves new problems through retrieval and reuse, which overcomes the difficulty that many knowledge and rules cannot be acquired in RBR. The CBR matches the current situation to a problem successfully solved in the past to get answers or inspiration. In the process of matching, the solution of the historical case is modified to better adapt to the specific situation of the current problem.

Due to the complexity of traffic accident formation and responsibility allocation, accident handling needs rich professional knowledge and experience which cannot be expressed simply by mathematical model. Case-based Reasoning (CBR) has become a hot research topic.

\section{Related Work}

The research on intelligent handling of traffic accidents in foreign countries is relatively early. In the 1970s, the NSA of the United States used computer to assist traffic police in analyzing traffic accidents and produced a series of analysis software, such as Simulation Model of Automobile Collision Program (SMAC), Calspan Reconstruction of Accident Speed on the Highway Program (CRASH3), Engineering Dynamics Corporation Reconstruction of Accident Sneed on the Highway Program (EDCRASH). At the same time, the developed countries also continue to develop accident cause analysis software to provide assistance in the handling of traffic accidents.

Many research groups are devoted to the research of CBR theory and methods, such as the Artificial Intelligence and Knowledge Systems Research Group of Kaiserslautern University funded by the German government, the Intelligent Decision Assistance Research Group of the NAVAL Intelligence Laboratory of the United States, etc. Our country also has extensive research on the application of expert system in traffic accident handling. Professor Li et al [6]. put the knowledge and experience of the traffic accident handling expert system into the computer in the form of knowledge base, and imitated the procedures and principles of the law enforcement to deal with the accident, to reason about the violation of the law and determine the responsibility of the litigants, and finally formed the relevant accident documents. Tu et al [7]. developed an intelligent decision support system for traffic accident handling, which applied the knowledge in the field of artificial intelligence to the identification of liability for traffic accidents.

However, the focus of the above research direction is to solve the similar analogy relationship problem between cases, and less attention is paid to the intelligent identification of the primary liability and secondary liability of the accident litigants in similar cases. In China's judicial practice, the litigants pay more attention to who should be mainly responsible for the occurrence of accidents, so as to determine who should compensate for the casualties and property losses caused by accidents.

Therefore, this paper proposes a traffic accident intelligent responsibility identification system based on Casebased Reasoning and D-S Evidence Theory. In the process of liability identification, Case-based Reasoning and D-S Evidence Theory are combined to analyze cases in case database. According to the case similarity, the basic probability of the litigants bearing the main liability is fused to obtain the probability interval of each litigant bearing the main liability, so as to determine the main liability person of the accident.

\section{System Model}

\subsection{Overall System Architecture}

In order to realize the scientific decision of "same case, same judgment" and distinguish the main liability subject and secondary liability subject in traffic accident, this road traffic accident liability identification system is designed. The road traffic accident liability identification system relies on the traffic accident handling case database, including intelligent retrieval of similar cases in traffic accidents and probability integration of main liability by D-S Evidence Theory, as shown in Fig. 1. 


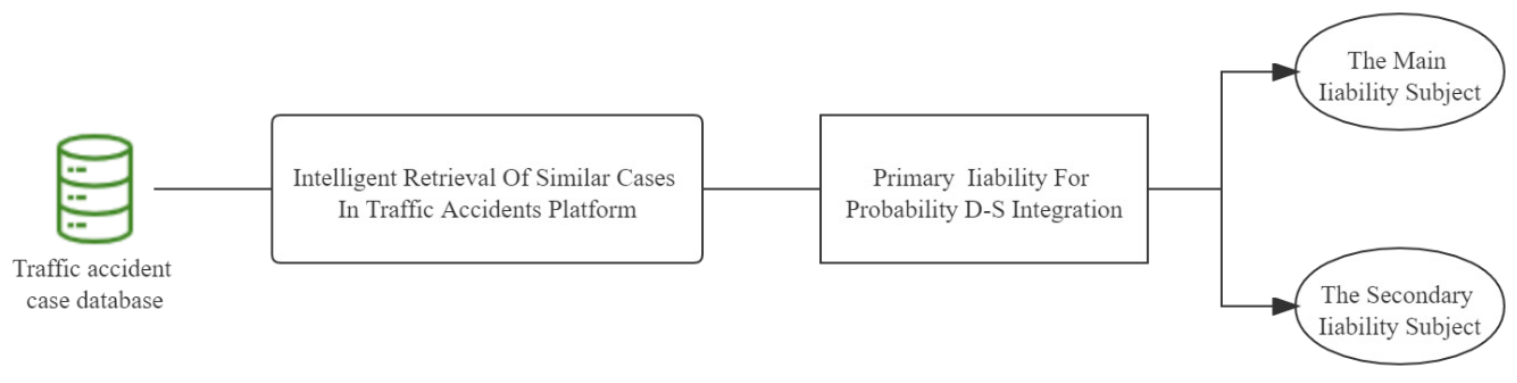

Fig. 1. Road traffic accident liability identification system architecture

According to the behavior type and other attributes, the intelligent retrieval of similar cases platform matches the historical cases with high similarity with the new cases, filtrates the top cases with the highest similarity as a reference, and gives the similarity of each case.

D-S Evidence Theory primary liability probability fusion is that according to the similarity of similar cases, the probability of the litigants bearing the main responsibility is given. Then, Dempster evidence is synthesized for the probability judgment of different cases by using D-S evidence theory to generate the reliability function and likelihood function of the litigants bearing the main responsibility, and generate the probability interval of the litigants bearing the main responsibility. Finally, the main liability subject and the secondary liability subject of the accident are determined by comparing the main liability probability interval of different litigants.

\subsection{Intelligent Retrieval of Similar Cases in Traffic Accidents}

\subsubsection{Case Representation}

The judgment ability of the accident liability identification system is positively related to the "knowledge" of the system, the more "knowledge" the system has, the more accurate the system will be in the accident liability identification. Therefore, in order to achieve artificial intelligence, it is necessary to store "knowledge" in a certain form in the computer.

The knowledge representation process is the process of encoding knowledge into some data structure. The same knowledge can be represented in many different ways, and different representations may produce different effects. In case-based reasoning [8-9] expert system, the main knowledge is represented in the form of case structure description [10-11]. Based on the characteristics of road traffic accident handling system, Framework Representation Method is adopted to represent "knowledge".

When a similar situation occurs, Framework Theory does not need to re-analyze the problem to construct the frame, but instead looks for the remembered frame and uses the details of the new event to supplement the contents of the frame. A framework is a data structure that describes a fixed situation and can generally be thought of as a network composed of nodes and relationships. The highest level of the framework is fixed, and it describes what is always true for a hypothetical situation. A framework can contain a variety of information: information describing things, information about how to use the framework, information about what is expected to happen next and what to do if the expected event does not happen, etc. This information is contained in the various slots or sides of the framework.

The idea of establishing the framework is consistent with the idea of accident processing by experts, so it is suitable for the knowledge representation of accident processing systems based on CBR reasoning techniques. The case representation in the traffic accident liability identification system is based on the traffic accident register, and the features required in case processing are obtained by summarizing a large number of cases. As an example, introduce the case representation of the road traffic liability identification system for a traffic accident involving a car and a pedestrian, as shown in Fig. 2. 


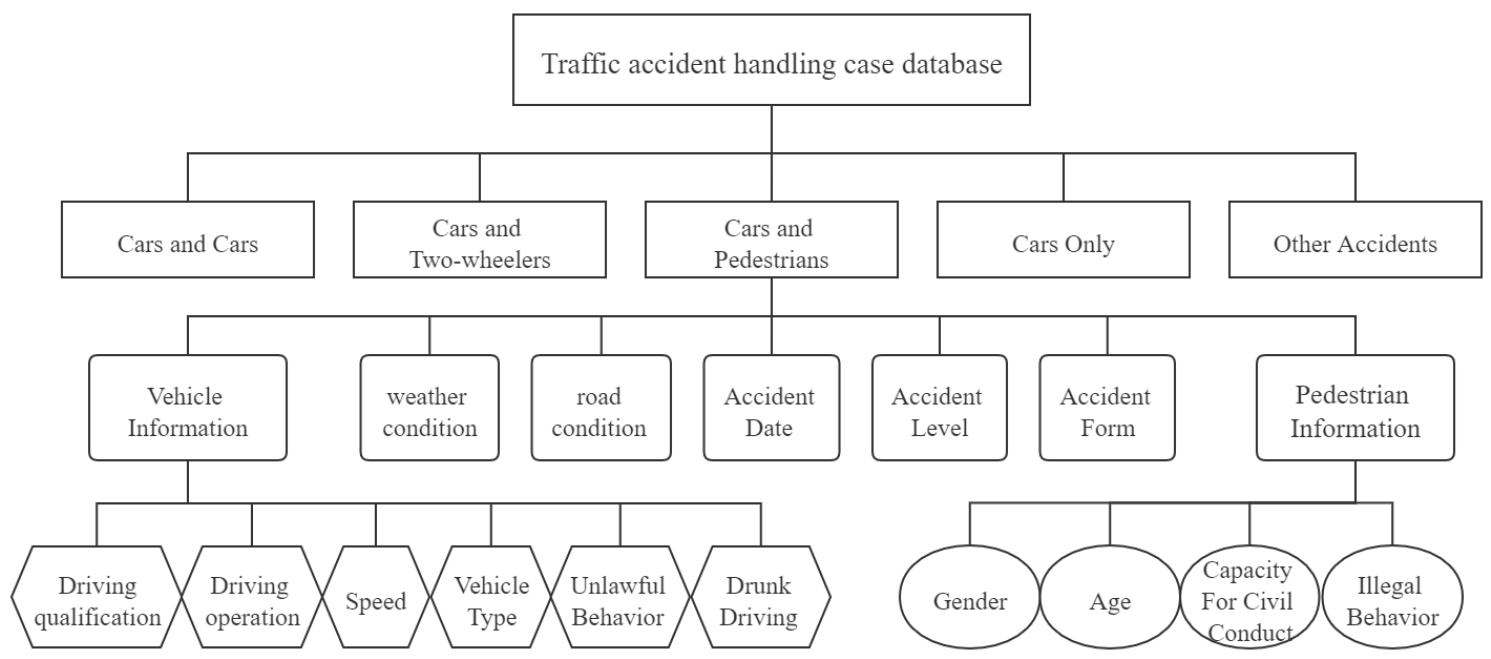

Fig. 2. Case expression of a traffic accident involving vehicles and pedestrians

\subsubsection{Structure of the Case Library}

The organization structure and storage of cases are important issues of the case base, which is currently organized and stored mainly in the form of a database. As mentioned above, the organization of the case base and the representation of the cases are related. The system represents "knowledge" in the form of a framework, and the organization of the case base is described in Fig. 3.

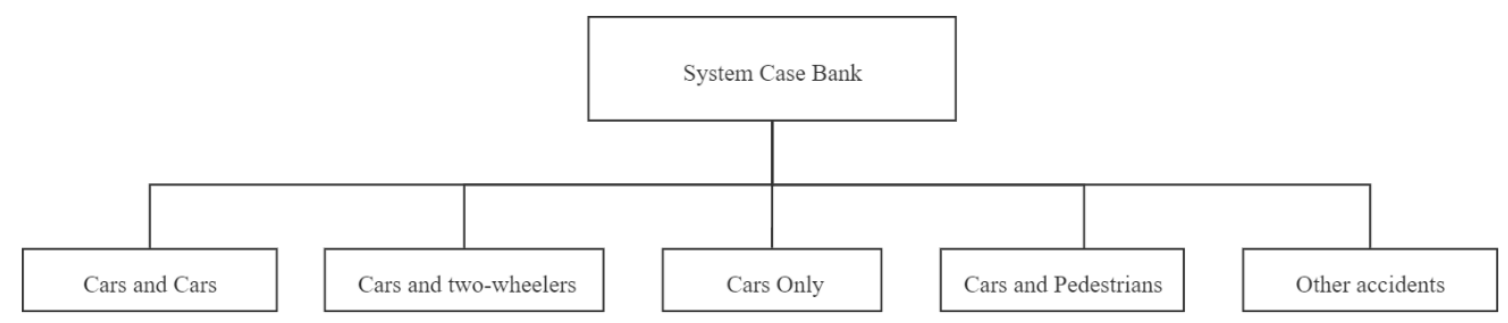

Fig. 3. Organizational structure of case base

Before the construction of the traffic accident case base, cases are represented in a variety of forms, which may be text, images, etc. They share a common characteristic of being raw and unprocessed. The case database can be constructed only after the common features are extracted from these case data. When these features are represented in the form of a frame, they are represented in the form of a slot. Thus, the main frame and the slots correspond to the table names and data items in the database. When the slots are expanded as sub-frames, the subframes and sub-features correspond to other tables in the database. These tables and the corresponding tables of the main frame form the relationship of the tables in the database, which are normalized to obtain specific units in the case base by using the data specification theory in the database. Therefore, by populating data items, cases are stored in the case library in the form of tables and represented as data mainly.

\subsubsection{Case Retrieval and Matching in the Accident Liability System}

After establishing the knowledge representation method, the key to the success of a reasoning system is concentrated in two aspects: one is the speed of case retrieval, and the other is the accuracy of case retrieval. The accident handling system deals with accidents based on case-based reasoning. The retrieval algorithm for finding similar cases is the key issue. The selection of retrieval algorithm involves the following aspects:

(1) The attributes of traffic accident cases. The characteristics and properties inherent in things in the real world 
are called attributes [12]. For the traffic liability identification system, the attributes of the case include the speed of the car, road conditions, running a red light, accident level, weather conditions, driver status, etc. For different classification methods, the attributes of cases can be divided into different categories. Attributes can be divided into two categories according to whether there are definite rules or not. By categorizing the case attributes, the optimal solution can be quickly retrieved according to the case attribute category during case retrieval.

(2) The threshold value in the accident liability identification system. Through the introduction of the concept of threshold value in the traffic liability identification system, the new cases put into the system are compared with the cases in the case database. If the distance between them is less than the threshold value, the cases are similar. The threshold value can be used to get a result set in the case database. The larger the threshold value, the larger the result set. On the contrary, the smaller the threshold value, the smaller the result set. The result must exist in the result set. Threshold is mainly used to divide the boundary of retrieval results and speed up the judgment of retrieval results. In the actual retrieval process, the determination of threshold value needs many times of comparison to determine the optimal solution.

(3) The basic weight of the case attribute in the liability identification system. Generally, the coefficients of linear polynomials are called weight, and the matrix composed of coefficients is called weight vector. The weight vector plays an important role in the identification of similar cases. In the accident liability identification system, judgments are made based on the attributes of the case. The weight vector reflects the importance of each attribute to the event. For example, when using the nearest neighbor method to judge the similarity of accident cases, the similar relationship between traffic accidents $A i$ and $A r$ is:

$$
\mathrm{S}\left(A_{i}, A_{r}\right)=\sum_{j=1}^{n}\left(W_{j} \times \mathrm{S}_{j}\left(a_{i j}, a_{r j}\right)\right) W_{j}
$$

$W j$ is the characteristic weight of a certain attribute of the case; $n$ is the number of attributes of the traffic accident case.

\subsubsection{Implementation of Case Retrieval Algorithm}

This system is based on case-based reasoning, using a case matching method that combines hierarchical filtering and K-nearest neighbor algorithm. The process of case filtrating is shown in Fig. 4.

Each attribute of the accident case should constitute a representation with the attribute of the case in the case database. Therefore, a number axis is used for characterization. Each case in the case base determines an attribute interval jointly by the threshold and attribute weights. The same attribute distribution interval of each case can have intersection. Before the case retrieval, the case attributes in the case library should be in the form of $\mathrm{N}$ number axes.

The case search of traffic accidents is particularly special. In the accident liability, the number of litigants and the form of the accident have more influence on the search results. Therefore, the number of litigants is matched first, and the classified case database is directly called. And then the accident type is matched in the case database.

Initial Case Library

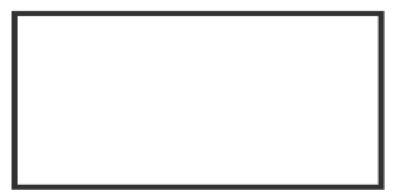

Post-Filtering Case Library

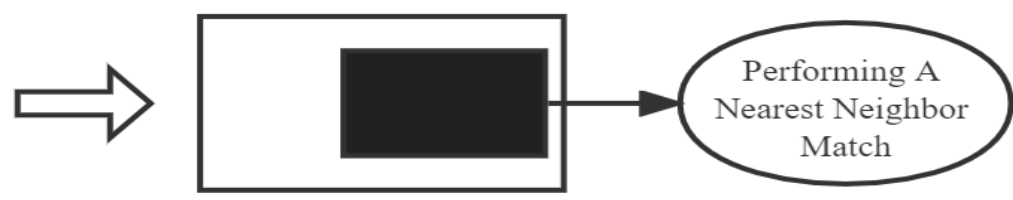

Fig. 4. The process of case filtrating

According to a specific order, the attributes of the new case are compared and analyzed with the attributes of the cases in the case library. After the non-matching cases are excluded step by step, the similar cases are obtained. In turn, the efficiency of retrieval is improved.

When matching cases according to the above method, the distribution of corresponding attributes is also different due to the different weights of each attribute of the case. If the comparison is done in the rising order of weight, there may be cases where there is a big difference in the intermediate results, which will affect the final 
result. Therefore, in this system, the above problems can be avoided to a certain extent by comparing the weights in descending order. In this way, we can get a relatively small number of cases containing a set of solutions. Since the set has undergone preliminary filtrating and the number is relatively small, the K-nearest neighbor algorithm can be used to calculate the similarity to find the $\mathrm{M}$ cases with the highest similarity. The flowchart of case matching is shown in Fig. 5.

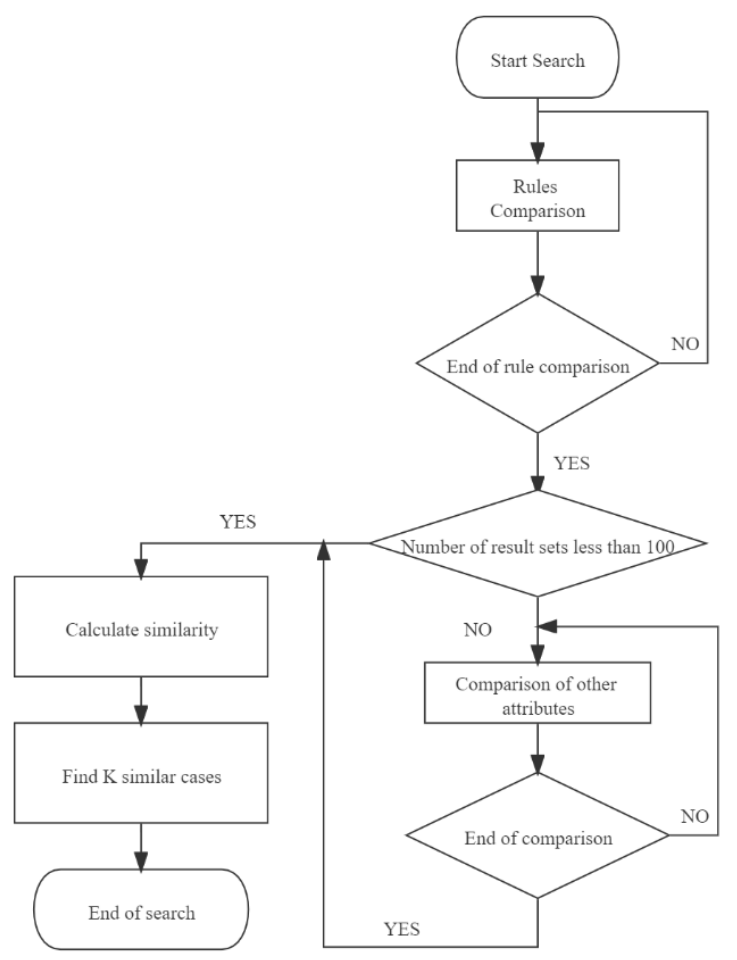

Fig. 5. Structure of case matching process

The entire case matching process is as follows:

(1) The new case and the $\mathrm{N}$ cases in the case library are used for rule reasoning. There are multiple rules in the rule set. We stipulate that the first rule is: the accident vehicle type and the number of litigants in the case. Calling the corresponding case sub-library after judging the vehicle type and the number of litigants. After filtering for each rule, the filtering process ends. At this stage, the $\mathrm{K}$ value is selected to be 1 , and the extreme value filtering is performed. Similar cases are filtered in the first layer to shorten the filtering time.

(2) Judge according to the number of result sets after filtering in step (1). If the number is less than 100, go to step (4), if the number is more than 100 , go to step (3).

(3) Filter and compare other attributes of the new case until the number of result sets is less than 100.

(4) Compare the attributes of the new case with the remaining cases in the case library, and save the result in the form of a matrix:

$$
\delta=\left[\begin{array}{cccc}
\delta_{11} & \delta_{12} & \cdots & \delta_{1 n} \\
\cdots & \delta_{i j} & \cdots & \cdots
\end{array}\right]
$$

Where $i=1,2, \ldots, m, j=1,2, \ldots, n$. Where $i$ represents the $i$-th case and $j$ represents the $j$-th attribute. $\delta_{i j}$ represents the local similarity.

(5) Multiply the weight vector with the above matrix to obtain the similarity between the new case and each case in the case library. The formula is as follows: 


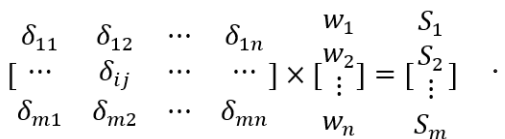

(6) According to the result of step (5), the $M$ cases with the greatest similarity are selected as the result.

\subsection{Primary Responsibility for Probability D-S Integration}

The occurrence of traffic accidents is affected by multiple factors such as weather, road conditions, models, drivers' driving habits, unreasonable laws and regulations, and traffic control, resulting in different degrees of casualties and property losses. It is difficult to find a historical case in which the accident environment, the subject of the accident, the cause of the accident and the degree of damage are identical. It is not scientific to take the liability determination result of the case with the highest similarity as the basis of the determination of the new case directly.

D-S Evidence Theory is a complete theory dealing with uncertain problems, which uses Dempster's synthesis rule to fuse multiple subjects [13-15]. For new traffic accidents, the probability that the litigants bear primary liability is an uncertain data. Case similarity can be defined as the probability that the similar liable person bears the main responsibility and D-S Evidence Theory is used to fuse the similarity of cases with the highest similarity. Thus, the probability interval of the main responsibility of each accident subject is determined, and the responsibility is assigned more scientifically.

When a traffic accident occurs, the person who has a direct relationship with the traffic accident is listed as an accident litigant. The mathematical set composed by litigants is $N=\left\{N_{1}, N_{2}, \ldots, N_{i}, \ldots, N_{n}\right\}$ which constitutes the $N$ whole domain of traffic accidents litigants. The mathematical set of similar cases filtrated according to the traffic accident case library is $X=\left\{X_{1}, X_{2}, \ldots, X_{j}, X_{m}\right\}$. Among them, case similarity $S_{1} \geq S_{2} \geq \ldots \geq S_{m}$. According to the classification of accident liability in real life, there are $n+1$ hypotheses for the determination of the main responsible person: "Litigant $N_{i}$ is the main responsible person" and "The main responsible person cannot be determined". Then, the mathematical set of all hypothesis of the problem is $\varphi=\left\{\varphi_{1}, \varphi_{2}, \ldots, \varphi_{i}, \ldots, \varphi_{n}, \varphi_{n+1}\right\}$. Where $\varphi_{i}=\left\{N_{i}\right\}$ indicates that "Litigant $N_{i}$ is mainly responsible", $1 \leq i \leq n ; \varphi_{n+1}=\left\{N_{1}, N_{2}, \ldots, N_{i}, \ldots, N_{n}\right\}$ indicates the hypothesis that "it cannot be determined who is primarily responsible". In case $X_{j}$, when the behavior of the main liable person is highly similar to that of the litigant $N_{i}$, we define the similarity of case $X_{j}$ as the probability that the litigant $N_{i}$ bears the main responsibility. That is, the probability that the hypothesis $\varphi_{i},, 1 \leq i \leq n+1$, being true is denoted as $P_{i, j}$; The probability of determining the validity of other hypotheses except $\varphi_{i}$ in the same similar case is denoted as $1-P_{i, j} / n$. In the same case, the sum of the basic probabilities of different hypothesis in $\varphi$ whole domain is 1 . Cases $X_{1}, X_{2}, \ldots, X_{n}$ respectively give the basic probability distribution (BPA) shown in Table 1 .

Table 1. Basic probability distribution in similar cases

\begin{tabular}{cccccc}
\hline & $X_{1}$ & $X_{2}$ & $\cdots$ & $X_{n}$ & $\cdots$ \\
\hline$\varphi_{1}$ & $P_{1,1}$ & $\frac{1-P_{2,2}}{n}$ & $\cdots$ & $\frac{1-P_{n, n}}{n}$ & $\cdots$ \\
$\varphi_{2}$ & $\frac{1-P_{1,1}}{n}$ & $P_{2,2}$ & $\cdots$ & $\frac{1-P_{n, n}}{n}$ & $\cdots$ \\
$\vdots$ & $\vdots$ & $\vdots$ & $\cdots$ & $\vdots$ & $\cdots$ \\
$\varphi_{n}$ & $\frac{1-P_{1,1}}{n}$ & $\frac{1-P_{2,2}}{n}$ & $\cdots$ & $P_{n, n}$ & $\cdots$ \\
$\varphi_{n+1}$ & $\frac{1-P_{1,1}}{n}$ & $\frac{1-P_{2,2}}{n}$ & $\cdots$ & $\frac{1-P_{n, n}}{n}$ & $\cdots$ \\
\hline
\end{tabular}

In the process of distributing the basic probability of each hypothesis in $\varphi$ whole domain, the basic probability distribution function mathematical set of each similar case is defined as $h(x)=\left\{h_{l}(x), h_{2}(x), \ldots, h m(x)\right\}$. We can see that $h(x)$ satisfies $h(\phi)=0$ and $\sum \varphi_{i \in U} h\left(\varphi_{i}\right)=1$.U is an identification framework. If $h\left(\varphi_{i}\right)>0$, then $\varphi_{i}$ is called the focal element.

The reliability function of some hypothesis $\varphi_{i}$ refers to the sum of the probabilities of all subsets of the hypothesis:

$$
\operatorname{Bel}\left(\varphi_{i}\right)=\sum_{B \in \varphi_{i}} m(B)
$$


The likelihood function of some hypothesis $\varphi_{i}$ refers to the sum of the probabilities whose intersection with the hypothesis is not empty:

$$
P l\left(\varphi_{i}\right)=\sum_{B \cap \varphi_{i} \neq \varnothing} m(B)
$$

For hypothesis $\varphi_{i}$, the closed interval $\left[\operatorname{Bel}\left(\varphi_{i}\right), \operatorname{Pl}\left(\varphi_{i}\right)\right]$ composed of reliability function and likelihood function is the confidence interval of hypothesis $\varphi_{i}$, which represents the confirmation degree of hypothesis $\varphi_{i}$.

For $\forall \varphi_{i} \in U$, Dempster's composition rule for mass functions $h_{1}, h_{2}, \ldots, h_{m}$ on identifying frame $U$ is:

$$
\left(h_{1} \oplus h_{2} \oplus \cdots \oplus h_{n}\right)\left(\varphi_{i}\right)=\frac{1}{K} \sum_{\varphi_{1} \cap \varphi_{2} \cap \cdots \cap \varphi_{n}=N_{i}} h_{1}\left(\varphi_{1}\right) \cdot h_{2}\left(\varphi_{2}\right) \cdots h_{n}\left(\varphi_{n}\right) \cdot
$$

Among,

$$
K=\sum_{\varphi_{1} \cap \varphi_{2} \cap \cdots \cap \varphi_{n} \neq \emptyset} h_{1}\left(\varphi_{1}\right) \cdot h_{2}\left(\varphi_{2}\right) \cdots h_{n}\left(\varphi_{n}\right)=1-\sum_{\varphi_{1} \cap \varphi_{2} \cap \cdots \cap \varphi_{n}=\emptyset} h_{1}\left(\varphi_{1}\right) \cdot h_{2}\left(\varphi_{2}\right) \cdots h_{n}\left(\varphi_{n}\right) \cdot
$$

$K$ is a normalized factor, and $1-K$ reflects the degree of conflict of evidence.

Then,

$$
\begin{gathered}
\operatorname{Bel}\left(\varphi_{n+1}\right)=P l\left(\varphi_{n+1}\right)=\left(h_{1} \oplus h_{2} \oplus \cdots \oplus h_{n}\right)\left(\varphi_{n+1}\right)=\frac{1}{K} \sum_{\varphi_{1} \cap \varphi_{2} \cap \cdots \cap \varphi_{n+1}=N} h_{1}\left(\varphi_{1}\right) \cdot h_{2}\left(\varphi_{2}\right) \cdots h_{n+1}\left(\varphi_{n+1}\right) . \\
\operatorname{Bel}\left(\varphi_{i}\right)=\frac{1}{K} \sum_{\varphi_{1} \cap \varphi_{2} \cap \cdots \cap \varphi_{n+1}=N_{i}} h_{1}\left(\varphi_{1}\right) \cdot h_{2}\left(\varphi_{2}\right) \cdots h_{n+1}\left(\varphi_{n+1}\right) \cdot \\
\operatorname{Pl}\left(\varphi_{i}\right)=\frac{1}{K} \sum_{\varphi_{1} \cap \varphi_{2} \cap \cdots \cap \varphi_{n+1}=N_{i}} \sum_{\varphi_{1} \cap \varphi_{2} \cap \cdots \cap \varphi_{n+1}=N_{i}} h_{1}\left(\varphi_{1}\right) \cdot h_{2}\left(\varphi_{2}\right) \cdots h_{n+1}\left(\varphi_{n+1}\right) .
\end{gathered}
$$

The probability interval of litigant $N_{i}$ bearing the main responsibility of the accident is:

$$
\left[\operatorname{Bel}\left(\varphi_{i}\right), P l\left(\varphi_{i}\right)\right]
$$

Similarly, we can use a sample method to calculate the probability interval for other litigants to bear the main liability. Thus, according to the probability interval of the main liability of each litigant in the traffic accident, the main liability subject and the secondary liability subject are determined.

\section{Simulation and Result Analysis}

The legal degree and reasonable degree of the liability distribution of the litigants involved in the traffic accident are defined as the Deterministic Level (DL) of the litigants' primary or secondary liability. We take the deterministic level of primary liability and secondary liability as the measurement index to analyze the model. We selected traffic accident cases from a province in China in the past three years (2018-2020) as the basic case database for case analysis, and selected some traffic accidents in the first half of 2021 in the province as test cases. Based on the primary liability identification model proposed in this paper, the litigants in the test case are distinguished from the primary liability and the secondary liability. We compare the DL of liability of litigants in test cases when traffic accident cases of different years are used as case base. Meanwhile, the influence of the number of litigants $N$ on the DL of liability is compared. 


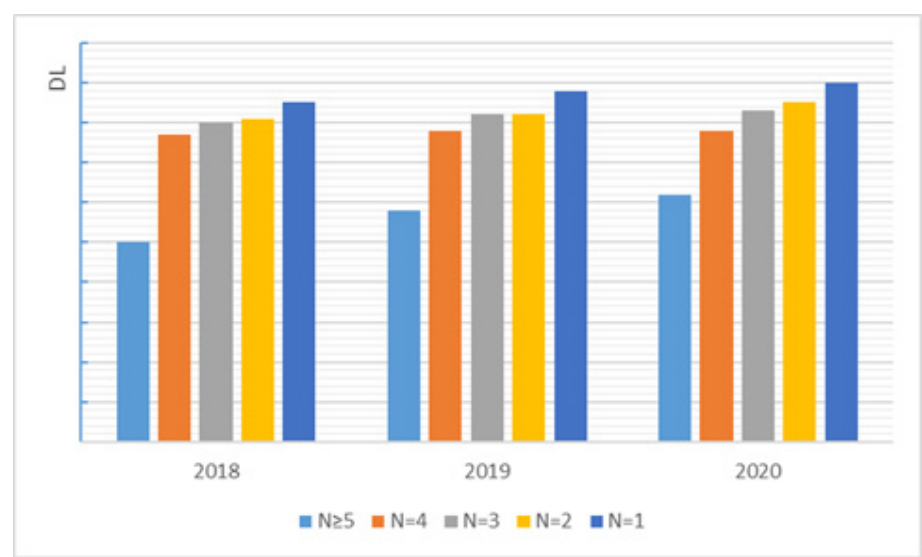

Fig. 6. The relationship between DL and the number of litigants and the time of case base

It can be seen from Fig. 6 that the DL of liability of litigants decreases with the increase of the number of litigants $N$. This is because when a traffic accident involves a large number of litigants, it generally forms a severe or extra-serious traffic accident. This kind of accident may be caused simply by one litigant's improper operation, or it may be affected by weather, natural disaster, damage to road traffic facilities, emergencies and other multiple factors. Therefore, the identification of traffic accident liability with a large number of litigants has great uncertainty, resulting in low efficiency of the model. But when the number of litigants is small $(N<5)$, the model shows excellent performance. It can also be seen that the model has a higher degree of DL about the liability of the litigants when training reasoning with case base close to time. We suspect that this has something to do with the new administrative regulations and judicial interpretations issued by the government in the process of social governance.

\section{Conclusion}

This paper proposes a traffic accident intelligent liability identification system based on Case-based Reasoning and D-S Evidence Theory. Case analysis was carried out by combining Case-based Reasoning and D-S Evidence Theory. According to the similarity of the case, the basic probability of the litigants bearing the main responsibility is fused, and the probability interval of the litigants' main responsibility is obtained, so as to determine the main responsible person of the accident. Through the practical example test, the method proposed in this paper has achieved remarkable results in the identification of the main liability of traffic accidents.

\section{Acknowledgement}

This work has been supported by the National Key Research and Development Program of China (Grant number 2018YFC0831900).

\section{References}

[1] H. Xiao, Research on Related Problems of Traffic Accident Confirmation, Hebei Law Science (12)(2018) 126-133.

[2] Q. Yuan, Y. Peng, X. Xu, X. Wang, Key points of investigation and analysis on traffic accidents involving intelligent vehicles, Transportation Safety and Environment (3)(4)(2021) 1-11.

[3] H. Liu, R.-C. Li, The Application of Fuzzy Set Pair Analysis Model for Identifying Liability for Injury in Traffic Accidents at Work, Journal of Lanzhou University of Finance and Economics 35(1)(2019) 42-49.

[4] K. Ivan, I. Haidu, J. Benedek, S.M. Ciobanu, Identification of traffic accident risk-prone areas under low-light conditions, Natural hazards and earth system sciences 15(9)(2015)191-197.

[5] W. Jun, Put it Another Way "Certificate of Liability for Traffic Accidents"-Analysis of Traffic Accident Damage Compensation Cases Only, Education Teaching Forum (2019).

[6] J. Li, G. Yu, W. Guan, et al., Preliminary Discussion on Expert System of Traffic Accident Settlement, Science and Technology of Highway Traffic 17(1)(2000) 51-54.

[7] R. Zhang, X. Tu, Intelligent Decision Support System for Traffic Accident Handling Based on CBR, Computer Engineering and Applications 38(2)(2002) 3. 
[8] A. Maalel, H. Hadj-Mabrouk, Contribution of Case Based Reasoning (CBR) in the Exploitation of Return of Experience. Application to Accident Scenarii in Railroad Transport, Computer Science (2012) arXiv: 1203.0656.

[9] I. Watson, Case-Based Reasoning is a Methodology not a Technology, Knowledge-Based Systems 12(5-6)(1999) 303-308.

[10]S. Sharma, D.H. Sleeman, REFINER: a case-based differential diagnosis aide for knowledge acquisition and knowledge refinement, in: Proc. of EWSL 88: Proceedings of the European Working Session on Learning, 1988.

[11]P. Cunningham, A. Bonzano, knowledge engineering in a real world case-based reasoning application (2017) 1-15.

[12]A. Aamodt, E. Plaza, Case-Based Reasoning: Foundational Issues, Methodological Variations, and System Approaches, Ai Communications 7(1)(1994) 39-59.

[13]X. Fan, M.J. Zuo, Fault diagnosis of machines based on D-S evidence theory. Part 1: D-S evidence theory and its improvement, Pattern Recognition Letters 27(5)(2006) 366-376.

[14]B. Chen, J. Wang, S. Chen, Prediction of pulsed GTAW penetration status based on BP neural network and D-S evidence theory information fusion, International Journal of Advanced Manufacturing Technology 48(1-4)(2010) 83-94.

[15]G. Zhao, A. Chen, G. Lu, et al., Data Fusion Algorithm Based on Fuzzy Sets and D-S Theory of Evidence, Journal of Tsinghua University: Natural Science (1)(2020) 8. 